\title{
PREFACE: MULTISCALE AND MULTIPHYSICS MODELING OF "COMPLEX" MATERIALS AND ENGINEERING APPLICATIONS
}

The present volume is a special issue of selected papers from the 11th edition of a special symposium session, "Multiscale and Multiphysics Modeling for Complex Materials," organized within the framework of the 9th International Conference on Computational Methods (ICCM2018), held in Rome, Italy, in August 2018, with Professor Trovalusci as chairman. The early focus of the symposium was to bridge the gap between solid mechanics and materials science, providing a forum for the presentation of fundamental, theoretical, experimental, and practical aspects of mechanical modeling of materials with complex microstructures and complex behavior.

Among various invited and contributing authors, the conference featured plenary lectures by Professors Somnath Ghosh, from Johns Hopkins University of Baltimore, Maryland, USA; Paulo B. Lourenço, of the University of Minho, Portugal; and Siegfried Schmauder, from the Institute for Materials Testing, Materials Science and Strength of Materials (IMWF), University of Stuttgart, Germany. All these lecturers also contributed to this special issue. The issue is divided into two separate volumes due to the number of contributions attracted. Globally, the whole issue collects fifteen articles by selected invited authors who participated in the mentioned symposium session as well as others. Each contribution has undergone a standard review process, and only papers which received positive recommendations from at least two international referees were included.

These contributions provide a survey of the multiscale approaches proposed to describe materials with various internal structures at different scales (nano/micro, meso, macro), from composite, to shape memory, to masonry-like materials, and with complex behaviors such as plasticity, damage, fracture, and phase transformation. Most of the papers aim at detecting the structural performances of advanced materials in various engineering applications ranging from microelectronics to civil engineering. The articles are briefly introduced in the paragraphs below.

Ghosh and Guo (2019) [1] develop a finite element model for coupled electromechanical systems. Transient dynamic simulations are performed by considering continuum damage relations, which are needed as a field-based virtual damage sensor. The code is validated by comparing with analytical results and those from commercial software. An electric field-based damage indicator function is proposed and calibrated from data obtained through numerical solutions. The function relates the electric field difference for undamaged and damaged conditions to the damage parameter, its rate, and mechanical and piezoelectric material properties. The virtual damage sensor is used to examine damage conditions in a stretchable piezoelectric serpentine conductor.

Lourenço and Silva (2020) [2] present a set of advanced models for the mechanical study of masonry, including the usual micromodeling approaches, macromodeling, and multiscale techniques. An extensive overview of its computational features is provided. The engineering application of such strategies is presented and covers problems starting from the masonry components level (mesoscale) to the structural element itself, and ultimately to the level of monumental buildings (super large). The structural safety assessment and/or strengthening schemes evaluated are performed amid the static, slow dynamics or earthquakes and fast dynamics or impact and blast ranges.

Haoyun et al. (2020) [3] adopt a 3D digital image correlation (DIC) system for investigating the fracture behavior of structural steel S355. The DIC system captures the crack propagation and strain field variation on the surface of a side grooved compact tension [C(T)] specimen. The 2D and 3D Gurson-Tvergaard-Needleman (GTN) model is used to simulate the ductile fracture of the specimen and the shape of the stable crack growth region. The DIC technique allows the strain variation and crack propagation on the specimen surface to be obtained. A comparison between experimental data and numerical simulation results shows that the GTN well describes the ductile fracture behavior of the C(T) specimen from S355.

The article by Spanos et al. (2020) [4] deals with the determination of the effective electrical and thermal conductivity of reinforced carbon nanotube (CNT) nanocomposites using a numerical percolation approach and Monte Carlo 
simulation. A nanocomposite representative volume element (RVE) is generated in a 3D spatial domain according to given input parameters. Connections among nanotubes are modeled using a spanning network algorithm. Interconnected nanotubes are converted into equivalent resistor networks to determine the effective electrical or thermal conductivity. Statistical variation at each volume fraction of nanotube filler is taken into account via Monte Carlo simulations. The effect of various microstructural parameters is investigated, the RVE size is optimized, and results are validated by comparison to experimental data from the literature.

In the article by Barretta et al. (2020) [5], a strain gradient model based on both Eringen's and Lam's nonlocal modified strain gradient formulation is provided. The present theoretical framework is based on three scale parameters which describe nonlocality, dilatation, and stretch gradient. It has been shown that these parameters significantly affect the response of one-dimensional nanorods. The problem is formulated through a variationally consistent approach based on suitably selected test fields, projected for formulating well-posed static and dynamic problems of engineering interest. Exact solutions are provided in terms of nonlocal and gradient characteristic parameters. Validation of the proposed strategy is carried out by comparing the contributed results with those obtained by the modified nonlocal strain gradient theory.

The work by Pisano et al. (2020) [6] represents a contribution in the field of nonlocal elasticity theory for the description of size effects in small-scale beams. The work is focused on the extension of a strain-difference-based nonlocal elasticity theory, previously developed by the authors, to the case of shear-deformable beams by incorporating a parametric warping model. The formulation covers a variety of beam modeling assumptions, ranging from the Euler-Bernoulli beam to the Timoshenko beam as limiting cases. The boundary value problem equations are solved by a set of eight uncoupled auxiliary integral equations independent of the boundary conditions. Numerical solutions for simple beam problems are reported with particular concern to size effects and to their sensitivity to shear deformation.

In the paper by Aminpour and Rizzi (2020) [7], a one-dimensional continuum model endowed with internal structure is proposed as a coarse descriptor of the necking of graphene nanostructures under tension in the nonlinear elasticity framework. The paper focuses on the postbuckling behavior of graphene sheets undergoing uniaxial tension loadings, but the procedure can be applied to other two-dimensional graphene nanostructures, as nanoribbons or nanotubes. The interatomic actions are described by the modified Morse potential, and the strain energy density of a RVE is given in terms of nanoscopic strain measures, expressed as a function of continuum strain measures thorough suitable relationships between the discrete and continuum kinematics. A perturbation analysis is performed to obtain the first bifurcation point on the trivial equilibrium path, together with the initial tangent to the bifurcated path. A peculiar postbuckling behavior is detected and discussed.

The work by Mazzucco et al. (2020) [8] discusses a multilevel procedure, characterized by the adoption of laser scanner techniques combined with CAD, X-ray computed tomography, and a particle random disposition algorithm. Such a framework reproduces an exact finite element model of concrete at the mesoscale in terms of particles packing and representation of material heterogeneities, together with a nonlinear constitutive characterization developed within the framework of nonassociated plasticity coupled with damage. The approach is tested on a typical application of structural failure in confined conditions under extreme mechanical loads.

Formica et al. (2020) [9] propose an optimization of the storage modulus and hysteretic damping capacity of carbon nanotube (CNT) nanocomposites. The analysis is proposed via a differential evolution algorithm coupled with an ad hoc finite element implementation of a nonlinear 3D mesoscale theory. Such theory describes the hysteresis due to the shear stick-slip between the nanotubes and the hosting matrix. The optimization problem seeks to determine the set of material parameters of a multilayer stacking sequence that can give rise to the largest storage modulus and damping capacity of the nanocomposite. The results confirm that the genetic-type multilayer nanocomposite damping optimization can be an effective design method.

In the work by Addessi et al. (2020) [10], a multiscale model for the analysis of the in-plane response of periodic masonry walls is presented. The overall constitutive behavior of the composite material is derived through a homogenization procedure based on the transformation field analysis (TFA) extended to the case of interfaces. A nonlinear constitutive law accounting for damage and friction phenomena is considered for joints, whereas a linear elastic constitutive relationship is assumed for the blocks. The proposed multiscale procedure is implemented into a finite element code. Validation examples are carried out: First, the response is analyzed comparing results obtained with the presented homogenization procedure with those recovered by detailed nonlinear finite element analysis. Second, the 
structural behavior of masonry panels subjected to compression-shear loads is studied. The reliability and numerical accuracy of the proposed approach are shown by providing different validation examples, referring to both unit-cell and structural scales.

The paper by Callari and Froiio (2020) [11] focuses on simulating the localization of propagating internal erosion in rigid porous media. The interaction between backward erosion piping and soil movement is also modeled. Exchange and transport of solid and fluid mass equations in a saturated poro-elasto-plastic solid embedding a propagating erosion conduit conveying a multiphase flow is analyzed. A numerical finite element framework was set up to solve the related coupled equations. The performance of the proposed formulation is assessed through an analysis of the soil response to the progressive dewatering of a cofferdam. The hydromechanical coupling and the ability to capture both the onset and propagation of piping are crucial for an interpretation of the limiting states typically observed in these structures.

Li et al. (2020) [12] present the framework of a second-order concurrent computational homogenization method for saturated granular materials. A staggered discrete element method (DEM) / finite element method (FEM) solution scheme is proposed for nonlinear hydromechanical analysis of saturated discrete particle assembly. The effective stress measures with the elastoplastic modular tensor, volume averages of pore pressures, and Darcy's velocities with the permeability coefficient tensor are determined and upscaled to a high scale. A FEM-(DEM/FEM) nested solution scheme is devised for the proposed second-order concurrent computational homogenization method. Numerical examples and results demonstrate the capabilities and performances of the proposed method in multiscale modeling of hydromechanical behaviors, particularly, softening and localization behaviors occurring in saturated granular structures.

The work by Chisari et al. (2020) [13] deals with the structural response of masonry structures under extreme loading conditions, such as seismic events. A multiscale approach is proposed in which an accurate mesoscale model, accounting for the specific masonry bonds, is utilized in virtual tests for the calibration of material parameters of a macroscale model, assuming energy equivalence between the two scales. The proposed methodology is applied to a case study considering the results obtained in previous experimental tests on masonry components subjected to cyclic loading and on a masonry building under pseudodynamic conditions to represent earthquake loading. The results confirm the potential of the proposed approach, computationally attractive compared to other homogenization techniques, and highlight some critical issues, such as the importance of selecting appropriate virtual tests for model calibration, which can significantly influence accuracy and robustness.

In the paper by Fantuzzi et al. (2020) [14], advantages of micropolar theory in modeling anisotropic composite materials with local rigid microstructure are discussed. This study focuses on composites made of hexagonal rigid particles which interact among themselves through elastic interfaces so that the deformation energy of the material is concentrated only at the interfaces. Three particle geometries are investigated, such as orthotetragonal, auxetic, and chiral. Novel results have been achieved by presenting the behavior of panels with various material symmetries under localized loads.

Huang et al. (2020) [15] present a multiphysics and multiscale design strategy. First fluid flow is coupled with heat transfer in both fluids and solids. The component is optimized for performance with such integrated multiphysics coupled models. Then, the optimized component is modeled only for its structural reliability using a "top-down" approach that begins from an elastic solution, followed by a more detailed and coupled elastoplastic continuum model for the critical zone, and then finally modeled at the microscale to reveal the effects of the material microstructure. A design example is shown for a modular plasma-facing component in a fusion energy device (plasma divertor). The cooled unit has a microengineered tungsten foam protective tile on top of solid tungsten closed tubing with heat removal internals.

We hope these research issues will provide opportunities to identify and discuss future developments in the field of multiscale modeling and design of advanced materials.

\section{ACKNOWLEDGMENTS}

Our special thanks go to the editor-in-chief, Jacob L. Fish, for inviting us to select and collect papers for this special issue and for assisting us in the editing work. P. Trovalusci acknowledges the support of PRIN 201 Grant, project

Volume 18, Issue 1, 2020 
2017HFPKZY (B86J16002300001) and Sapienza Research Grants "Progetti Medi” 2017 (B83C17001440005) and "Progetti Grandi" 2018 (B81G19000060005).

Guest Editor:

Patrizia Trovalusci

Department of Structural Engineering and Geotechnics

Sapienza University of Rome

Via Gramsci 53, 00197 Rome, Italy

Co-Guest Editors:

Nicholas Fantuzzi

Department of Civil, Chemical, Environmental and Materials Engineering

University of Bologna

Viale del Risorgimento 2, 40136 Bologna, Italy

Maria Laura De Bellis

Department of Engineering and Geology

University "G. D' Annunzio" of Chieti and Pescara

Viale Pindaro 42, 65127 Pescara, Italy

\section{ARTICLES IN THIS ISSUE}

1. Ghosh, S. and Guo, S., Developing a Virtual Damage Sensor Using a Coupled Electro-Mechanical Fe Model of a Piezoelectric Material.

2. Lourenço, P.B. and Silva, L.C., Computational Applications in Masonry Structures: From the Meso-Scale to the SuperLarge/Super-Complex.

3. Haoyun, T.H., Schmauder, S., Li, Y., and Zhou, H., Digital Image Correlation Tracking and Gurson-Tvergaard-Needleman Modelling of Crack Propagation on Steel S355.

4. Spanos, P., Higginson, C., Ketron, T., and Roy, A., 3A Resistor Network Computational Model for the Determination of Electrical and Thermal Properties of Nanocomposites.

5. Barretta, R., Faghidian, A., Marotti de Sciarra, F., and Pinnola, F.P., On Nonlocal Lam Strain Gradient Mechanics of Elastic Rods.

6. Pisano, A.A., Fuschi, P., and Polizzotto, C., A Strain-Difference based Nonlocal Elasticity Theory for Small-Scale ShearDeformable Beams with Parametric Warping.

7. Aminpour, H. and Rizzi, N.L., On the Necking of Graphene Nanostructures.

8. Mazzucco, G., Pomaro, B., Xotta, G., Salomoni, V.A., and Majorana, C.E., An Efficient Geometric Reconstruction of Mesoscale Concrete Structures Accounting for Confinement Scenarios.

9. Formica, G., Milicchio, F., and Lacarbonara, W., Storage and Damping Optimization in Hysteretic Multilayer Nanocomposites.

10. Addessi, A., Gatta, C., Marfia, S., and Sacco, E., Multiscale Analysis of In-Plane Masonry Walls Accounting for Degradation and Frictional Effects.

11. Callari, C. and Froiio, F., A Hydro-Mechanical Finite Element Formulation for Localized Internal Erosion in Porous Media, with Application to Backward Piping in Cofferdams.

12. Li, X., Zhang, S., and Duan, Q., Second-Order Concurrent Computational Homogenization Method and Multi-Scale HydroMechanical Modeling for Saturated Granular Materials. 
13. Chisari, C., Macorini, L., and Izzuddin, B.A., Multiscale Model Calibration by Inverse Analysis for Nonlinear Simulation of Masonry Structures under Earthquake Loading.

14. Fantuzzi, N., Trovalusci, P., and Luciano, R., Multiscale Analysis of Anisotropic Materials with Hexagonal Microstructure as Micro-Polar Continua.

15. Huang, Y., Nadvornick, W., Ghazari, A., and Ghoniem, N., Multiphysics-Multiscale Modeling of Plasma-Facing Structures in Extreme Heat and Radiation Environments.

Volume 18, Issue 1, 2020 